\title{
Further confirmation that spiking of intravenous bags does not cause time-dependent microbial contamination
}

\author{
Scott Segal MD, MHCM \\ Department of Anesthesiology, Wake Forest University School of Medicine, Winston-Salem, North Carolina
}

To the Editor-I read with interest the paper by Brock-Utne et $\mathrm{al}^{1}$ on the microbial stability of spiked bags of intravenous fluid without the use of International Standards Organization level 5 (ISO-5) conditions, in contradistinction to one interpretation of the requirements of United States Pharmacopeia (USP) Chapter 797. It was certainly encouraging to see that bags prepared in an ordinary anesthesia workroom did not show microbial contamination in their hands. I present here related data from a study in which anesthesia technicians prepared IV fluid bags either in the open anesthesia workroom or under an ISO-5 hood.

\section{Methods}

In this study, 1-L bags of lactated Ringer's $(\mathrm{N}=80)$ were spiked by anesthesia technicians with conventional intravenous tubing sets used in the operating suite. The anesthesia workroom was located in the semirestricted area of the suite, and accordingly, caps and masks were worn during bag preparation. Sterile gloves and gowns were not worn. In total, 40 bags were prepared in the open workroom, and 40 bags were prepared under an ISO-5 hood located in the workroom. Fluid was run through the tubing set, the roller clamp was actuated to stop flow, and the sterile end cap of the tubing set was replaced. Ten bags in each group were sampled at time zero (immediately after preparation), and at 1 hour, 12 hours, and 24 hours after preparation. Samples were obtained by removing the sterile end cap and extracting $5 \mathrm{~mL}$ into a sterile container, which was then drawn into a sterile syringe and injected into standard blood culture bottles for automated detection of microbial growth (VersaTREK, TREK Diagnostics, Cleveland, OH). Cultures were incubated for up to 5 days, and any detectable growth underwent Gram staining and subculturing to identify the organism. ${ }^{2}$ Each bag was sampled at only 1 time point to avoid contamination during the fluid extraction. Positive controls were samples deliberately contaminated with hand flora during sampling, and all demonstrated positive growth.

\section{Results}

The culture results are shown in Table 1 as the number of contaminated bags $(\mathrm{n})$ divided by the total number of samples $(\mathrm{N})$. One bag from each group was positive $(P>>.05)$. Both

Author for correspondence: Scott Segal, MD, MHCM, Department of Anesthesiology, Wake Forest School of Medicine, Winston-Salem, NC. E-mail: bsegal@wakehealth.edu

Cite this article: Segal S. (2019) Further confirmation that spiking of intravenous bags does not cause time-dependent microbial contamination. Infection Control \& Hospital Epidemiology 2019, 40, 111-112. doi: 10.1017/ice.2018.282
Table 1. Culture Results

\begin{tabular}{lcc}
\hline $\begin{array}{l}\text { Time of Sampling } \\
\text { (Preparation Time) }\end{array}$ & $\begin{array}{c}\text { Control } \\
\text { (Workroom) }\end{array}$ & ISO-5 Hood \\
\hline 0 & $0 / 10$ & $0 / 10$ \\
\hline $1 \mathrm{~h}$ & $\begin{array}{r}1 / 10 \text { Propionibacterium } \\
\text { acnes }\end{array}$ & $0 / 10$ \\
\hline $12 \mathrm{~h}$ & $0 / 10$ & $0 / 10$ \\
\hline $24 \mathrm{~h}$ & $0 / 10$ & $\begin{array}{c}1 / 10 \text { Coagulase } \\
\text { negative Staphylococcus sp }\end{array}$ \\
\hline
\end{tabular}

contaminants were normal, generally nonpathogenic contaminants-most likely skin flora.

\section{Discussion}

Our results are consistent with those of Brock-Utne et al in that contamination did not clearly vary with time since preparation and was rare overall. In addition, there was no discernable effect of using an ISO-5 hood on rates of contamination. Notably, the only contaminated bag in the control (workroom) condition was detected 1 hour after preparation, which would have been compatible with administration under USP 797 regulations. ${ }^{3}$ We detected a low but nonzero rate of contamination, in contrast to Brock-Utne et al, who found none. However, it is likely that the absolute concentration of bacteria was very low and probably not pathogenic. Other work from our department has demonstrated that contamination of anesthesia syringes is rare, and when it occurs it is of vary low intensity, typically 100 colony-forming units $(\mathrm{CFU}) / \mathrm{mL}$ (data not shown). This rate is at least several orders of magnitude less than that observed almost universally during dental procedures, which almost never produce clinically important bacteremia. ${ }^{4,5}$ Similarly, dialysis patients are exposed to $\sim 100 \mathrm{~L}$ of dialysate solution per session, which may contain up to $100 \mathrm{CFU} / \mathrm{mL}^{6}{ }^{6}$

In summary, I agree that strict adherence to the 1-hour requirement in USP 797 regarding preparation of IV fluid infusion bags for anesthesia care is not supported by data demonstrating risk of bacterial infection. Furthermore, the use of laminar flow hoods does not appear to materially affect the rate of contamination.

Acknowledgments. The author wishes to express gratitude to the anesthesia technicians and microbiology laboratory personnel who assisted with this study.

Financial support. No financial support was provided relevant to this article. 
Conflicts of interest. The author reports no conflicts of interest relevant to this article.

\section{References}

1. Brock-Utne JG, Smith SC, Banaei N, Chang SC, Alejandro-Harper D, Jaffe RA. Spiking of intravenous bags does not cause time-dependent microbial contamination: a preliminary report. Infect Control Hosp Epidemiol 2018;39:1029-1030

2. Mirrett S, Hanson KE, Reller LB. Controlled clinical comparison of VersaTREK and BacT/ALERT blood culture systems. J Clin Microbiol 2007;45:299-302.
3. United States Pharmacopeial Convention. USP 27, NF 22: The United States Pharmacopeia, the National Formulary, Supplement 1. United States Pharmacopeial Convention; 2004:3121-3138.

4. Heimdahl A, Hall G, Hedberg M, et al. Detection and quantitation by lysisfiltration of bacteremia after different oral surgical procedures. J Clin Microbiol 1990;28:2205-2209.

5. Limeres Posse J, Alvarez Fernandez M, Fernandez Feijoo J, et al. Intravenous amoxicillin/clavulanate for the prevention of bacteraemia following dental procedures: a randomized clinical trial. J Antimicrob Chemother 2016;71:2022-2030.

6. Upadhyay A, Jaber BL. We use impure water to make dialysate for hemodialysis. Semin Dial 2016;29:297-299.

\title{
Re-estimating annual deaths due to multidrug-resistant organism infections
}

\author{
Jason P. Burnham $\mathrm{MD}^{1}$, Margaret A. Olsen PhD, MPH ${ }^{1}$ and Marin H. Kollef MD \\ ${ }^{1}$ Division of Infectious Diseases, Washington University School of Medicine, St Louis, Missouri and ${ }^{2}$ Division of Pulmonary and Critical Care Medicine, \\ Washington University School of Medicine, St. Louis, Missouri
}

To the Editor-Multidrug-resistant organisms (MDROs) are responsible for an increasing number of infections each year. ${ }^{1}$ An oft-cited statistic is that MDRO infections cause $>2$ million illnesses and 23,000 deaths each year in the United States. ${ }^{1}$ However, the true burden of MDRO infections remains uncertain due to insufficient national reporting rates and an absence of ICD-10 codes specifically for MDRO infections. Therefore, we sought to provide an updated estimate of deaths due to MDRO infections in the United States. Based on availability of data, we provide an estimation of mortality from MDROs for the year 2010 .

In 2010 in the United States, 2,468,435 deaths were registered, ${ }^{2}$ of which $715,000(29.0 \%)$ were inpatient hospital deaths. ${ }^{3}$ Using a conservative estimate for deaths due to sepsis, ${ }^{4} 34.4 \%$ of inpatient deaths occur among patients with sepsis, ${ }^{5}$ and the reported rate of MDR pathogens in sepsis is $28.8 \%{ }^{6}$ With these parameters, MDRO sepsis could cause 70,837 inpatient deaths each year (Table 1).

We have yet to assign infection as a cause of death to the $1,753,435$ outpatient deaths in 2010. Subtracting deaths that are unlikely to be due to infection (eg, suicides, accidents, and homicides), we are left with 1,572,624 deaths. After subtracting deaths due to infections, $1,550,536$ deaths remain. If $17 \%-19 \%$ of these 1,550,536 deaths are due to infections (despite not being indexed as such $)^{7}$ and $28.8 \%$ are due to MDROs, there would be $285,680-316,690$ deaths in which infection contributed to death, of which 82,276-91,207 deaths were due to MDROs (Table 1).

Summing the lower bounds of our estimates for inpatient and outpatient deaths due to MDRO infections gives us

Author for correspondence: Jason P. Burnham, Division of Infectious Diseases, Washington University School of Medicine, 4523 Clayton Avenue, Campus Box 8051, St Louis, MO 63110. E-mail: burnham@wustl.edu

Cite this article: Burnham JP, et al. (2019). Re-estimating annual deaths due to multidrug-resistant organism infections. Infection Control \& Hospital Epidemiology 2019, 40, 112-113. doi: 10.1017/ice.2018.304
Table 1. Estimates of Number of Deaths Due to Infection and MultidrugResistant Organisms in the United States in 2010

\begin{tabular}{lcc}
\hline Category & Deaths Due to Infection & $\begin{array}{c}\text { Deaths Due to Multidrug- } \\
\text { Resistant Organism Infection }\end{array}$ \\
\hline Inpatient & 245,960 & 70,837 \\
\hline Outpatient & $285,680-316,690$ & $82,276-91,207$ \\
\hline Total & $531,640-562,650$ & $153,113-162,044$ \\
\hline
\end{tabular}

153,113 deaths, a number almost 7 -fold higher than that estimated by the CDC (ie, 23,000). ${ }^{1}$ Assuming a worst-case scenario and summing the upper limits of our estimate, 162,044 deaths in the United States were due to MDRO infections in 2010 (Table 1). This would move MDRO infections to the third highest cause of death in the United States for 2010. All infections would then supersede the MDRO infections group as the third highest cause of death, ${ }^{2}$ with $>500,000$ (Table 1 ).

Our estimates illustrate a need for better surveillance and reporting mechanisms for MDRO infections. With rampant overuse of antibiotics, establishment of MDRO breeding and transmission centers (long-term acute-care hospitals and nursing facilities), and increasing rates of iatrogenic immunosuppression, the population at risk for MDRO infections and the likelihood of drug resistance will continue to increase. To address this critical issue, establishing the burden of MDROs is crucial to guide research funding allocation.

\section{Acknowledgments}

Financial support. Dr Kollef's efforts were supported by the BarnesJewish Hospital Foundation. Dr Burnham reports that this publication was made possible by the NIH-National Center for Advancing Translational Sciences (NCATS grant no. UL1 TR002345, subaward KL2 TR002346), 